\title{
Excision of Wilms' Tumor With Atrial Extension Under Moderate Hypothermia and Cerebral Perfusion
}

\author{
CRISTIAN BULESCU ${ }^{1}$, REMI DUBOIS ${ }^{2}$, FREDERIC HAMEURY ${ }^{2}$ and ROLAND HENAINE ${ }^{1}$ \\ ${ }^{1}$ Department of Adult and Child Cardiovascular Surgery and Heart Transplantation, \\ Louis Pradel Cardiologic Hospital, Bron, France; \\ ${ }^{2}$ Department of Urology, Visceral, Thoracic and Transplant Surgery, \\ Woman-Mother-Child Hospital, Bron, France
}

\begin{abstract}
Background: Wilms' tumor is the most common pediatric renal tumor. Almost half of all cases have involvement of the inferior vena cava, which must be addressed at the time of surgical excision. Further extension into the right atrium may pose an immediate vital risk and necessitates special operative techniques that employ cardiopulmonary bypass. Case Report: We report the case of a child with a left Wilms' tumor with inferior caval and right atrial involvement, which led to significant hemodynamic compromise and urgent surgery. A left nephrectomy and cavoatrial thrombectomy were performed via a sterno-laparotomy. Our strategy employed moderate hypothermic circulatory arrest at $26^{\circ} \mathrm{C}$ and antegrade cerebral perfusion in order to improve visualization and ensure complete thrombectomy and protection of the abdominal organs. Conclusion: This case emphasizes the advantages of moderate hypothermic circulatory arrest compared to deep hypothermic circulatory arrest and normothemic cardiopulmonary bypass.
\end{abstract}

Wilms' tumor is the most frequent renal tumor in childhood and is associated with a large frequency (4-10\% of patients) of vascular tumoral involvement (inferior vena cava and right atrium) (1,2); cavo-atrial extension has an increased risk of acute heart failure due to obstruction of flow through the tricuspid valve or pulmonary embolism.

Treatment for tumors at this stage involves nephrectomy and cavo-atrial thrombectomy using cardiopulmonary bypass, with or without hypothermic circulatory arrest. Although deep

This article is freely accessible online.

Correspondence to: Cristian Bulescu, 10 Alecu Russo street, 020523 Bucharest, Romania. Tel: +40 722763795, e-mail: cristianbulescu@gmail.com

Key Words: Wilms' tumor, hypothermia, antegrade cerebral perfusion. hypothermic circulatory arrest (DHCA) ensures a dry surgical field and end-organ preservation, it increases the risk of bleeding due to the combined effects of prolonged cardiopulmonary by-pass (CPB), hypothermia, platelet dysfunction and consumption of clotting factors. Conversely, normothermic, full-flow CPB with significant venous return from the inferior vena cava (IVC) does not allow an optimal visualization and increases the risk of incomplete resection of thrombus.

To reduce these risks, we have employed a strategy of moderate hypothermic circulatory arrest (MHCA) at $26^{\circ} \mathrm{C}$ with antegrade cerebral perfusion in a patient with a left Wilms' tumor, using superior vena cava (SVC) and infrarenal IVC cannulation.

\section{Case Report}

A 16-month-old boy, weighing $11 \mathrm{~kg}$, presented with nausea and an abdominal mass. Computed tomography (Figure 1) was performed and showed a $14 \times 12 \times 9 \mathrm{~cm}$ mass encompassing the left kidney, with complete thrombosis of the left renal vein. The thrombus extended into the IVC and the right atrium (RA), prolapsing through the tricuspid valve. The suprahepatic veins were partially occupied by thrombi. There were no secondary findings in the lungs, liver or contralateral kidney.

Based on these findings, a diagnosis of nephroblastoma was established. The patient was started on a four-dose chemotherapy regimen (vincristine-actinomycin); surgical resection was scheduled after the fourth dose. After the third dose of vincristine, he developed a septic condition with subsequent increase in the size of the atrial thrombus and obstruction of tricuspid antegrade flow. An urgent surgical resection was thus performed.

Surgical technique. A midline skin incision was performed from the sternal notch to the pubis, followed by a midline sternotomy. The abdominal part of the operation was performed first. 
The left genital vessels, urether and renal pedicle were completely dissected and transected. The renal tumor was mobilized and removed en bloc with the left adrenal gland and the entire periaortic ganglionic mass. Next, the IVC was dissected and mobilized up to the diaphragm, together with the origin of the suprahepatic veins. The hepatic pedicle was snared and two maneuvers of hepatic conditioning were performed.

The pericardium was opened. CPB was instituted between one aortic cannula, one SVC cannula and another angled-tip cannula placed in the infrarenal IVC. A left heart vent was inserted. The intrapericardial IVC was completely freed from its diaphragmatic attachments. The core temperature was reduced to $26^{\circ} \mathrm{C}$ and the aortic cannula was pushed into the innominate artery; MHCA was thus started. The aorta was cross-clamped and antegrade cold crystalloid cardioplegia was administered.

The right atrium was incised from the appendage down to the anterior aspect of the IVC. The intracardiac part of the tumor was about $4 \mathrm{~cm}$ in length; it was adherent to the right atrial wall, and was prolapsing through the tricuspid valve during systole, without being attached to valvular tissue. The tumor was successfully dissected away from the cardiac wall and the supradiaphragmatic portion of the IVC (Figure 2).

A lateral cavotomy was performed on the abdominal IVC, starting at the stump of the left renal vein, in a cephalad direction. The thrombus was easily separated from the vein wall and we succeeded in removing it in one piece, pushing it from the IVC to the RA (Figure 3). We further removed some thrombi from the suprahepatic veins.

The right atrium and the cavotomy were closed with running sutures. The arterial cannula was repositioned in the ascending aorta. Full-flow $\mathrm{CPB}$ was reinstated and the patient was rewarmed to $36^{\circ} \mathrm{C}$.

Separation from CPB was performed uneventfully. The MHCA time was $29 \mathrm{~min}$, aortic cross clamp time was 30 min, and total CPB time was $86 \mathrm{~min}$.

The Intensive Care Unit stay was also uneventful, with rapid extubation. Postoperative bleeding was minimal. The echocardiogram showed a completely permeable IVC, no residual thrombi and a trivial tricuspid regurgitation.

The patient resumed the chemotherapy sessions 10 days after the surgery, with a total of 27 weeks of combined vincristine-actinomycin treatment, associated with a total regimen of 10.8 Gy of abdominal radiation therapy. Nine months after the end of the adjuvant treatment, he is in an excellent clinical condition and without abdominal or cardiac echographic findings of tumoral recurrence.

\section{Discussion}

Complete surgical resections in association with chemo- and radiation therapy are essential for the long-term survival of patients with Wilms' tumor (3). For patients with stage III and
IV disease, the resection must also address the thrombi in the suprahepatic veins, IVC or RA; there are several surgical strategies available. Their goals are: Optimal visibility (a bloodless IVC) in order to ensure complete thrombectomy, restoration of a permeable IVC, and preservation of abdominal organs, mainly the liver and kidneys.

Some surgical strategies do not use CPB and rely on the complete mobilization of the IVC and 'milking' of the thrombus into the abdominal IVC (4) or pushing the thrombus down into the IVC from within a purse string placed on the right atrial appendage (5). The IVC is crossclamped above and below the thrombus and a cavotomy is performed, followed by excision of the thrombus. The Pringle maneuver (cross-clamping the hepatic pedicle in order to stop the venous return from the suprahepatic veins) is often used.

The complications of this method are the abrupt decrease in venous return to the heart following IVC cross-clamping, and warm liver ischemia. Splenic rupture due to acute occlusion of the porta has also been reported (6). The time available for the resection is quite short, enough only for the cavotomy, thrombectomy and closure of the vein. More complex vascular procedures (which are sometimes necessary because the tumor might infiltrate the IVC wall), such as patch enlargement or partial IVC replacement with a conduit, would thus be marginally tolerated by the patient.

Normothermic CPB has been used with venous return ensured via one SVC cannula and one femoral or infrarenal IVC cannula (6). This reduces the blood return to the IVC once it is opened for the thrombectomy. However, the abdominal viscera organs which drain to the portal vein or IVC are still perfused, and blood flow through the suprahepatic veins can make adequate visualization difficult. Complications include hepatic and kidney injury due to warm ischemia, reaching an incidence of up to $40-60 \%$ in some series (7). Intraoperative transfusion requirement is significant $(7,8)$. Intraoperative deaths due to massive bleeding or pulmonary embolism have been reported (8-10).

DHCA at $18-20^{\circ} \mathrm{C}$ addresses all of the concerns of adequate visualization, complete thrombectomy, vascular wall restoration, and abdominal organ preservation. The risk of bleeding is increased because of prolonged CPB time (due to cooling and rewarming periods), hypothermia-induced platelet dysfunction and hemodilution, and consumption of clotting factors (11). This can have serious consequences in a patient with extended areas of abdominal and thoracic surgical dissection.

Moderate hypothermic circulatory arrest with antegrade cerebral perfusion is a strategy used for many years in congenital heart surgery involving the aortic arch. A core body temperature of $26-280 \mathrm{C}$ ensures a period of about 45 minutes of lower body circulatory arrest. The brain is perfused by the arterial cannula which is advanced through 


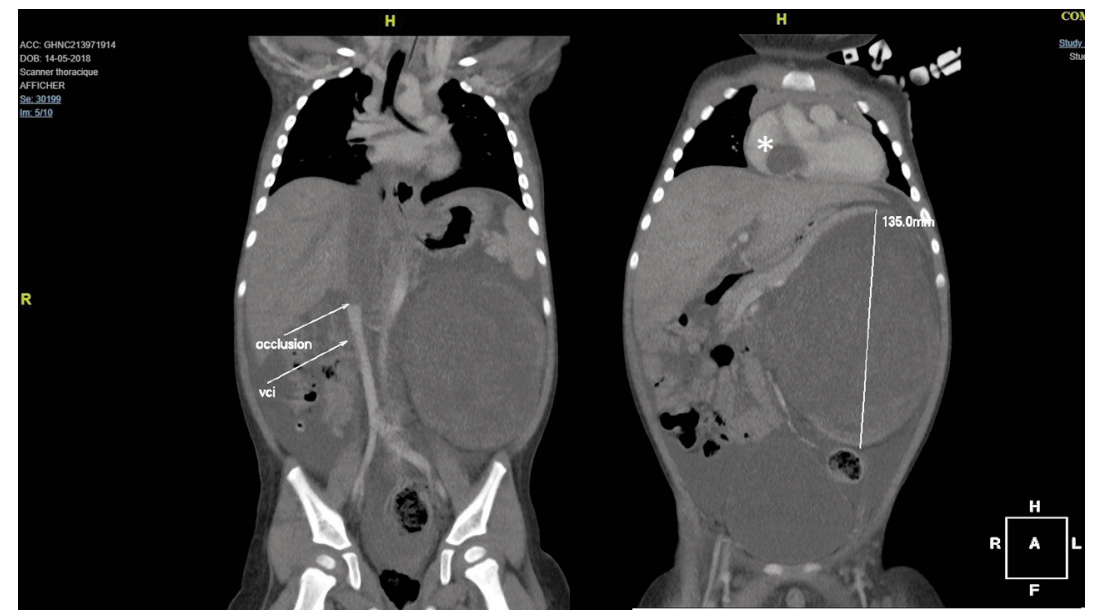

Figure 1. Computed tomographic scan of the infant patient prior to surgery. VCI: Inferior vena cava. Asterisk: Right atrial thrombus.

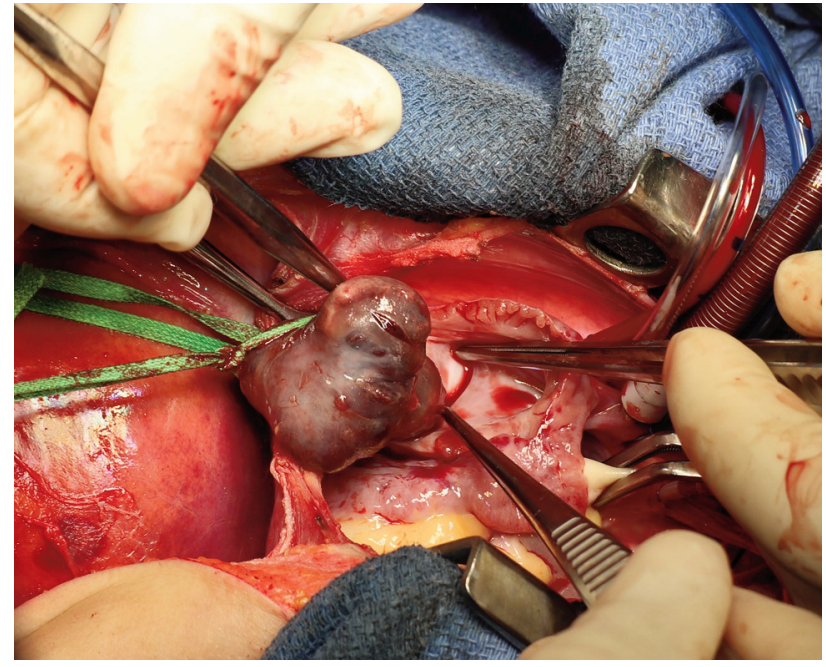

Figure 2. Surgical view. The atrial part of the thrombus was dissected from the right atrial wall.

the innominate artery into the right common carotid artery. There is no venous return from the abdominal viscera and there is a clear, dry field over the IVC and the suprahepatic veins. It also offers enough time for complex IVC reconstruction procedures, if needed.

As the abdominal IVC is already exposed during the procedure, full-flow CPB can be established using one SVC cannula and one infrarenal IVC cannula. This avoids the need for femoral venous cannulation, which cannot be employed in small children $(<20 \mathrm{~kg})$ due to their small vessels. Moreover, as the temperature gradient is lower

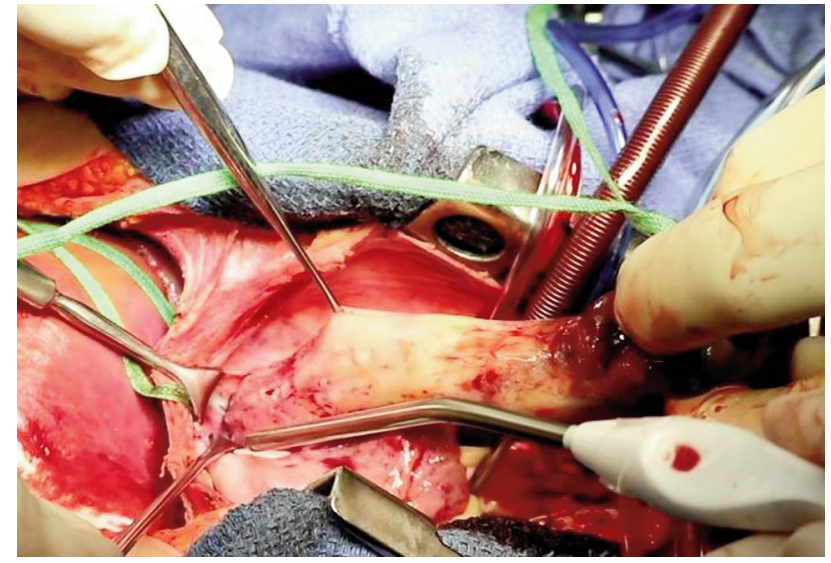

Figure 3. Surgical view. After inferior cavotomy and circumferential dissection of the thrombus, it was extracted in one piece from inside the right atrium.

compared to DHCA (being only $10^{\circ} \mathrm{C}$ ), the cooling and rewarming times are shorter, so the risk of bleeding is also greatly diminished.

In conclusion, we believe that the use of MHCA with antegrade cerebral perfusion is a very useful strategy for surgical thrombectomy in patients with stage IV nephroblastoma.

\section{Conflicts of Interest}

None declared. 


\section{Authors' Contributions}

All of the Authors have contributed to the writing and the revision of the article.

\section{References}

1 Szavay P, Luithle T, Semler O, Graf N and Fuchs J: Surgery of cavoatrial tumor thrombus in nephroblastoma: a report of the SIOP/GPOH study. Pediatr Blood Cancer 43(1): 40-45, 2004. PMID: 15170888 . DOI: $10.1002 /$ pbc. 20056

2 Al Diab A, Hirmas N, Almousa A, Abu-Hijlih R, Aljlouni F, Sultan I and Ghandour K: Inferior vena cava involvement in children with Wilms tumor. Pediatr Surg Int 33(5): 569-573, 2017. PMID: 28070651. DOI: 10.1007/s00383-016-4034-7

3 Irtan S, Ehrlich PF and Pritchard-Jones K: Wilms tumor: "Stateof-the-art" update, 2016. Semin Pediatr Surg 25(5): 250-256, 2016. PMID: 27955727. DOI: 10.1053/j.sempedsurg. 2016.09.003

4 Bader MI, Abdelaal K, Rogers T and Arul SG: A surgical approach to Wilms' tumour with retrohepatic vena caval extension. Pediatr Surg Int 29(3): 229-232, 2013. PMID: 23344152. DOI: 10.1007/s00383-013-3263-2

5 Patil MB, Montez J, Loh-Doyle J, Cai J, Skinner EC, Schuckman A, Thangathurai D, Skinner DG and Daneshmand S: Level III-IV inferior vena caval thrombectomy without cardiopulmonary bypass: long-term experience with intrapericardial control. J Urol 192(3): 682-688, 2014. PMID: 24704114. DOI: $10.1016 /$ j.juro.2014.03.112

6 Baniel J, Bihrle R, Wahle GR and Foster RS: Splenic rupture during occlusion of the porta hepatis in resection of tumors with vena caval extension. J Urol 151(4): 992-994, 1994. PMID: 8126847. DOI: $10.1016 / \mathrm{s} 0022-5347(17) 35145-5$
7 Stewart JR, Carey JA, McDougal WS, Merrill WH, Koch MO and Bender HW Jr: Cavoatrial tumor thrombectomy using cardiopulmonary bypass without circulatory arrest. Ann Thorac Surg 51(5): 717-21; discussion 721-2, 1991. PMID: 1850976.

8 Skinner DG, Pritchett TR, Lieskovsky G, Boyd SD and Stiles QR: Vena caval involvement by renal cell carcinoma. Surgical resection provides meaningful long-term survival. Ann Surg 210(3): 387-92; discussion 392-4, 1989. PMID: 2774709. DOI: 10.1097/00000658-198909000-00014

9 Chiappini B, Savini C, Marinelli G, Suarez SM, Di Eusanio M, Fiorani V and Pierangeli A: Cavoatrial tumor thrombus: singlestage surgical approach with profound hypothermia and circulatory arrest, including a review of the literature. J Thorac Cardiovasc Surg 124(4): 684-688, 2002. PMID: 12324725. DOI: $10.1067 / \mathrm{mtc} .2002 .124295$

10 Tsuji Y, Goto A, Hara I, Ataka K, Yamashita C, Okita Y and Kamidono S: Renal cell carcinoma with extension of tumor thrombus into the vena cava: surgical strategy and prognosis. J Vasc Surg 33(4): 789-796, 2001. PMID: 11296333. DOI: 10.1067/mva.2001.111996

11 De Robertis E, Kozek-Langenecker SA, Tufano R, Romano GM, Piazza $\mathrm{O}$ and Zito Marinosci G: Coagulopathy induced by acidosis, hypothermia and hypocalcaemia in severe bleeding. Minerva Anestesiol 81(1): 65-75, 2015. PMID: 24608516.

Received March 23, 2021

Revised May 5, 2021

Accepted May 7, 2021 